\title{
A Test Adaptation of the Modified Readiness for Inter-professional Learning Scale in Turkish
}

\author{
Arif Onan', Sevgi Turan', Melih Elcin', Nurettin Simsek², Kaan Zülfikar Deniz ${ }^{3}$ \\ ${ }^{1}$ Department of Medical Education and Informatics - Hacettepe University, Faculty of Medicine, Sihhiye Campus, 06100 Altindag, \\ Ankara, TURKEY. \\ ${ }^{2}$ Department of Computer Education \& Instructional Technology - Ankara University, Institute of Educational Sciences, 06590 Cebeci, \\ Ankara, TURKEY \\ ${ }^{3}$ Graduate School of Educational Sciences - Ankara University, Institute of Educational Sciences 06590 Cebeci, Ankara, TURKEY.
}

\begin{abstract}
Background: There is a need for healthcare professionals to develop teamwork and collaboration skills before they graduate. Inter-professional education is a suitable modality for these learning outcomes and it will be effective if it begins early in the undergraduate curriculum. Objectives: "Inter-professional Collaboration for Patient Safety" has been taught as an elective course in Hacettepe University. This new educational modality requires measuring tools to determine the qualifications of the learners and support the learning process. This study aimed to adapt the readiness for inter-professional learning scale (RIPLS) for use in Turkey. Methods: The guideline developed by Sousa and Rojjanasrirat (2011) guided the adaptation processes. The students enrolled in the study from Child Development, Dentistry, Medicine, Nursing, Nutrition and Dietetics, Paramedic, Pharmacy, Physiotherapy and Rehabilitation programs. Four hundred and eighty-four students completed RIPLS questionnaires. Psychometric properties were analysed by confirmatory factor analysis. Results: The results were consistent with the original scale. When Sousa's approach was followed: (i) improvements were needed for four items, (ii) the scale items were clear and understandable, (iii) the Turkish RIPLS presents good content validity, and (iv) the Turkish RIPLS has very good internal consistency $(0.87, n=484)$. Conclusions: The Turkish RIPLS can be used as a valid and reliable measurement tool for evaluating the RIPL. Adaptation studies showed that the RIPLS was sensitive to cultural context. Researchers should be cautious on performing cross-cultural comparisons of subscale 'roles and responsibilities' because The Turkish RIPLS had the lowest value of reliability for this subscale, like the original and the other adapted scales.
\end{abstract}

Key words: Inter-professional Learning, Readiness, Reliability, Validity, Scale Adaptation.

\section{INTRODUCTION}

There is a widespread consensus on the need for healthcare professionals to develop teamwork and collaboration skills for in order to deliver high-quality, effective, safe and sustainable health services. ${ }^{1-3}$ Changes in the methods of delivery in health services mandate different competencies for healthcare professionals, who need to be supported with continuous professional development and lifelong learning., ${ }^{4,5}$
Numerous studies reveal the importance and magnitude of the medical errors that occur during the delivery of healthcare. ${ }^{6-8}$ The World Health Organization (WHO) has reported that communication and coordination is the most important skill to develop to reduce patient suffering. ${ }^{9}$ As a result, an emphasis was placed on teamwork in healthcare and it was shown that effective teamwork increases service quality and reduces medical errors. ${ }^{10-12}$
Submission Date: 30-08-2016; Revision Date: 17-11-2016; Accepted Date: 23-11-2016

DOI: 10.5530/ijper.51.2.26 Correspondence: Arif Onan,

Hacettepe University, Faculty of Medicine,

Medical Education and Informatics, Sihhiye Campus Altindag Ankara, Turkey GSM: +90 5368512921 Phone: +90 3123052617 E-mail: aonan@hacettepe. edu.tr

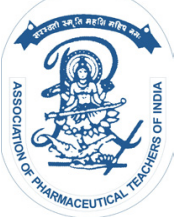

www.ijper.org 
Collaborative patient care practices require each healthcare professional to actively engage in patient care, interact with different healthcare professionals, organize for a common goal, respect the other professions' contributions to patient care, and get involved in decision-making processes. ${ }^{13}$ The developments and observed benefits of teamwork on patient safety led to the inclusion of these modalities in the education of future healthcare professionals. As the concept of inter-professional learning for inter-professional practice became widely adapted, the number of studies on the content and methods of teaching teamwork has increased.

There are various definitions for conceptualizing the collaborative learning of different professionals. In the present study, we used the term inter-professional learning/training for learning and promoting collaborative practice. ${ }^{14}$ The definition of inter-professional education that is widely accepted today was defined in 1997 as 'the occasions when two or more professions learn from and about one another to promote collaborative practice'. ${ }^{15}$

The principles of adult learning are utilized for the effective delivery of inter-professional education. ${ }^{4}$ Life experiences, prior knowledge, and readiness levels are all important in enriching the learning process and achieving the objectives of adult learning; put simply, learning can become easier when it is associated with experience. A special emphasis is placed on learning together in order for students to increase their abilities to work together; learning by doing is the best way to learn to work together. Seeing the world through the eyes of other professionals, creating a framework for the patient's problems, and finding possible solutions for those problems by considering the ideas of other professionals are the cornerstones of inter-professional learning. ${ }^{16}$

While debates exist on how to teach inter-professional learning skills, it is widely suggested that learning such skills begins early on, during undergraduate training. ${ }^{17}$ One of the most effective tools to teach these skills is to have students in different health professions spend time together during their undergraduate training. Various programs on inter-professional learning were developed with this principle in mind. The Canadian Inter-professional Health Collaborative Competencies Working Group. ${ }^{18}$ was published in 2010 and the American Inter-professional Education Collaborative Core Competencies ${ }^{19}$ were published in 2011. The $\mathrm{WHO}^{9}$ reviewed and published their patient safety training program in 2011.
Evaluation of the effectiveness of the teaching programs is paramount. Several challenges-organizational, structural, and attitudinal — can be present when these programs are executed, and the most difficult of these challenges is dealing with attitudes. ${ }^{20}$ Since it is expected that students will change their attitudes regarding inter-professional learning during their education, it is suggested that these changes are defined and supported prior to becoming an independent practitioner. ${ }^{21}$

The readiness of healthcare students for inter-professional learning scale (RIPLS) was developed by Parsell and Bligh $^{21}$ and has been widely used to evaluate interprofessional learning programs. Parsell and Bligh conducted a pilot survey of the newly developed RIPLS on a group of students from eight different professions. The 19-item RIPLS consisted of three subscales: teamwork and collaboration, professional identity, and roles and responsibilities. The internal consistencies (alpha coefficients) for the subscales were 0.88, 0.63, and 0.32 , respectively.

Despite the low degree of reliability in the last dimension in Parsell and Bligh's study, the RIPLS has continued to be used in the field. McFadyen et al. ${ }^{22}$ performed a validity and reliability study for RIPLS in order to answer these critiques a confirmatory factor analysis was conducted for the original 19-item scale. There were four subdimensions determined: teamwork and collaboration, negative professional identity, positive professional identity, and roles and responsibilities. McFadyen et al.22 reported the following Cronbach's Alpha values for these RIPLS sub-dimensions: $0.88,0.76,0.81$, and 0.43 , respectively. The results of the confirmatory factor analysis for those sub-dimensions were: GFI: 0.904, CFI: 0.942, RMSEA: 0.054, and Chi-square: 1.777. Since the confirmatory factor analysis results were acceptable in this test, the final version of the scale was accepted to have a more stable structure.

Numerous efforts have been made to adapt the RIPLS into different cultures and languages. The scale was adapted to the Swedish, ${ }^{23}$ Japanese, ${ }^{24}$ French, ${ }^{25}$ German, ${ }^{26}$ and Indonesian ${ }^{27}$ languages. Further validity and reliability studies were also conducted for the test in different languages and cultures.

Studies in inter-professional learning are relatively a new concept in Turkey and most efforts have been about promoting teamwork. The first educational program for developing inter-professional learning skills was implemented at Hacettepe University, named 'Interprofessional Collaboration for Patient Safety', and has been taught as an elective course since the 2013-2014 academic year. 
As mentioned above, it is critical to evaluate the effectiveness of training programs and monitor the progress of the inter-professional learning skill development of healthcare students throughout their education. Implementation and evaluation efforts were required to obtain an instrument for measuring attitudes about inter-professional education and collaborative practice. The primary purpose of this study was to conduct a test adaptation of RIPLS (modified by McFadyen et al).22 in Turkish to assess the readiness levels of healthcare professionals in inter-professional collaboration.

\section{MATERIALS AND METHODS}

\section{RIPLS questionnaire}

The modified scale was developed by McFadyen $e t$ al. ${ }^{22}$ using confirmatory factor analysis in a structural equations model. Cronbach's Alpha measurements were conducted for internal validity. Item correlation was confirmed in the final scale, which consisted of four factors and 19 items. The total Cronbach's Alpha value of the scale was 0.89. Necessary permissions for translating the scale were obtained through email communication; after obtaining permission, the adaptation process began.

\section{Translation and validation process}

The ethics committee approval was obtained from the Non-interventional Clinical Research Ethics Board of Hacettepe University (Date and number: 24.07.2013 GO 13/391 -10). The guideline developed by Sousa and Rojjanasrirat $^{28}$ led the translation, adaptation, and validation processes. The following actions were taken, as suggested by the guideline:

$1^{\text {st }}$ Step: Translation of the original scale into Turkish by a translation committee composed of two separate teams. Each one of these teams consisted of two English teachers and a subject expert. Two separate translations were then obtained.

$2^{\text {nd }}$ Step: Review of both translations by the translation committee and reaching consensus. Both sub-teams converged and reviewed the translation. The teams then reached consensus on the Turkish version of the scale.

$3^{\text {rd }}$ Step: Translation of the Turkish version of the scale back into English. The translation committee, in the $2^{\text {nd }}$ step, formed a subcommittee that consisted of an English teacher and a subject expert, who translated the scale back to English. The translation committee then reviewed and approved the translation.

$4^{\text {th }}$ Step: Comparison of the original scale with the one translated back to English from the Turkish version. This was done by the translation committee with special consideration given to the concept, meaning, and content of the adapted scale. The committee continued to review and make changes to the scale until a consensus was reached on the matching of both scales.

$5^{\text {th }}$ and $6^{\text {th }}$ Steps: Pilot test and psychometric evaluation of the initial version of the Turkish scale. Twenty bilingual (Turkish and English) medical school students were asked to evaluate the comprehensibility of the scale items and they were asked to rephrase the items in case they thought the items were not understandable. Cognitive evaluations of the students were considered during this process.

The initial version of the Turkish scale, which was fine-tuned after the pilot evaluation, was then reviewed by $11 \mathrm{PhD}$ members of the expert panel, which consisted of representatives from the departments of nutrition, child development, dentistry, pharmacy, physiotherapy, nursing, social services, paramedics, and medicine for concept and content; work continued until a consensus was reached. The panel board was also asked to group the scale items with the factors. The professional identity factors, positive and negative, were mentioned under professional identity, as in the original version. The panellists were not able to reach consensus on the grouping of the scale items within three factors. Therefore, the other sub-scales in the scale were grouped within three dimensions, as suggested by Parsell and Blight ${ }^{21}$ (teamwork and collaboration, professional identity, and roles and responsibilities).

The panel members made slight changes to statements \#10, 11, 16, 17, and 18 without changing the meaning. In Turkish culture, negative willingness or believes cannot be directly expressed. For this reason, Item 10 and 11 was changed to opinion and perspective statements. Those changes can be seen below:

- The statement 'I don't want to waste my time learning with other healthcare students' was changed to 'I think learning with other healthcare students is a waste of time' (Item 10).

- The statement 'It is not necessary for undergraduate healthcare students to learn together' was changed to 'I think learning from other healthcare students' experiences help my professional improvement' (Item 11).

- The statement 'Shared learning before qualification will help me become a better team worker' was changed to 'Shared learning with other healthcare students will help me to become an effective team worker', in order to better emphasize 'a better team worker' (Item 16).

- The statement ${ }^{\circ}$ The function of nurses and therapists is mainly to provide support for doctors' was 
changed to "The function of other healthcare workers is mainly to provide support for doctors', since our study was conducted with a variety of healthcare students and not just nurses, therapists, or doctors (Item 17).

- The statement 'I am not sure what my professional role will be' was changed to 'I am not sure about my professional role among healthcare workers', in order to better predict interprofessional collaboration (Item 18).

$7^{\text {th }}$ Step: Completion of all psychometric evaluations of the initial version of the Turkish scale by conducting a paper-based survey of the study group. The 5-point Likert scale survey was completed by 484 volunteer students from various healthcare professions during the spring semester of the 2013-2014 academic year. The descriptive characteristics and RIPLS scores of the students are given in Table 1.

\section{Statistical analysis}

Confirmatory Factor Analysis was employed to examine the model fit for each scale using multiple indices, including $\chi 2 / \mathrm{df}$, RMSEA, CFI, AGFI, RMR, SRMR, and NNFI. Cronbach's Alpha and Composite Reliability were calculated for internal consistency. The Lisrel (v 8.71) software was used to estimate structural equation modelling and IBM SPSS (v21) was used for other analyses. The items 10,12 and 17 were reverse scored.

\section{RESULTS}

Seven survey questionnaires were excluded from the study data since they had non-representative outliers. ${ }^{29}$ A total of 484 questionnaires were included in the study. The basic assumptions of multivariate statistics were all met in the data set.

\section{Factors}

The results obtained from the Confirmatory Factor Analysis (CFA) were consistent with the model presented by Parsell and Bligh $^{21}$ in the original version of the RIPLS. The Turkish version of the RIPLS consisted of 19 items. The sub-factor grouping was performed under three factors (Figure 1). Nine of the items were listed under the teamwork and collaboration factor, seven were under professional identity, and three under roles and responsibilities. The factor loadings obtained from factor analysis were statistically significant and numerically satisfactory except for Item 18 . The factors were all consistent with Parsell and Bligh's ${ }^{21}$ findings, allowing a comparative analysis (Table 2).

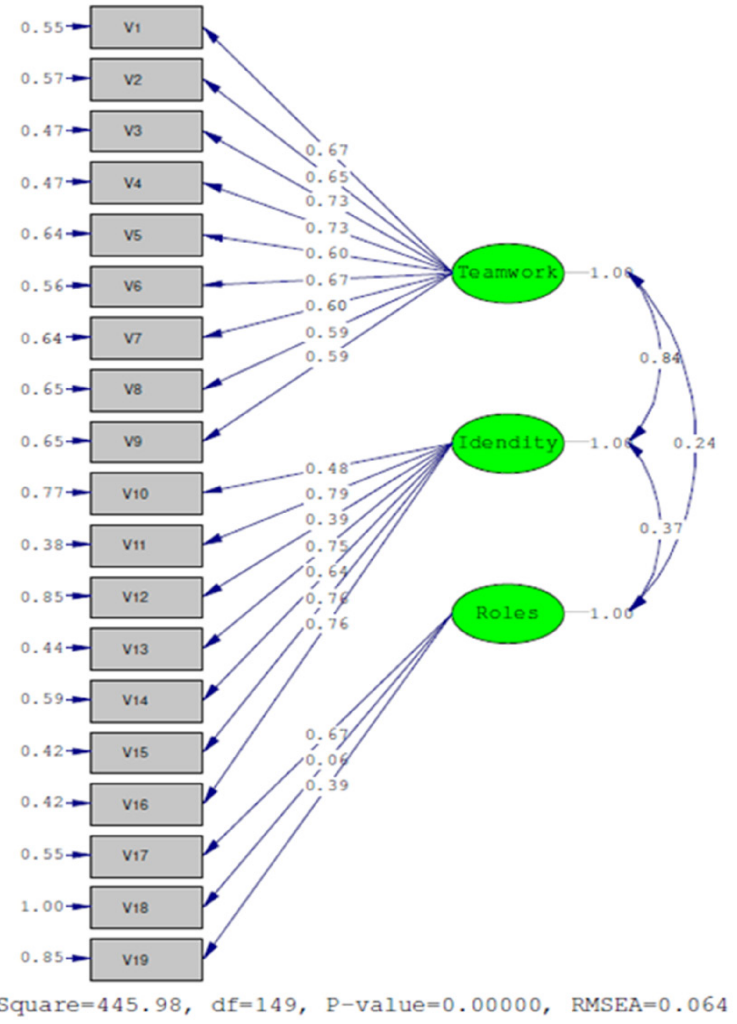

Figure 1: Structural equation modal of Turkish RIPLS.

Reliability

The overall Cronbach's Alpha value for the scale was 0.87. The individual Cronbach's Alpha values for the factors were $0.85,0.82$, and 0.28 , respectively. The Composite Reliability (CR) values of the factors were $\mathrm{CR}>0.70$ for the first two factors and $\mathrm{CR}<0.70$ for the last; these values were consistent with the Cronbach's Alpha values (Table 2). The Cronbach's Alpha and CR values of the first two factors met the reliability requirements for the first two factors; however, they failed to meet the same requirements for the third factor.

\section{Content validity}

The scale items were reviewed by 20 medical school students for ease of understanding prior to the study and their suggested corrections were made. A translation committee worked on the translation and comparison of the scale in order to have successful intercultural adaptation of the scale. Following this stage, the scale content was evaluated by another expert panel that represented nine different healthcare professions. Based on the reviews and critiques of the second expert panel, necessary changes were made in the scale in order to make the content easier to understand, given the cultural differences. 
The lowest level of item-level content validity index (I-CVI) was 0.818 and the highest level I-CVI was 1.000, while the average I-CVI was 0.914 . Similarly, the lowest expert proportion relevant for the scale items was 83.333, the highest was 100, and the scale-level index/ Average (S-CVI/Ave) value was 91.414. Content validity is also, in effect, tested by CFA, as CFA is a theorydriven method of analysis.

\section{Construct validity}

We used structural equation models (SEM) in our study, as the literature suggested, to test the predefined mental structures. Our results showed that the Turkish version of the RIPLS was indeed consistent with the original RIPLS developed by Parsell and Bligh ${ }^{21}$ (Figure 1).

The results of construct validity (convergent validity and discriminant validity), based on correlations among sub-dimensions and the average variance extraction (AVE), were satisfactory except for factor 3 (Table 3).

\section{DISCUSSION}

The confirmatory factor analysis results for RIPLS and its adaptation studies showed that the proposed models had a high degree of compatibility. The CFA values obtained during the adaptation studies of RIPLS are presented in Table 4. The index values obtained in the present study were higher than those obtained from other RIPLS adaptation studies; as a result, the structure of the tested Turkish version of the scale is thought to be consistent.

In the present study, after CFA, similar values were obtained with the structure suggested and confirmed by Parsell and Bligh ${ }^{21}$ and with those tested and confirmed by Lauffs et al. ${ }^{23}$ and Mahler et al. ${ }^{26}$ With the exception of Cloutier et al. ${ }^{25}$ the team and collaboration sub-scale in all studies was consistent with the results of Parsell and Bligh. ${ }^{21}$ The lowest degree of reliability was obtained in the roles and responsibilities sub-scale in the present study; similarly, Parsell's study also yielded low levels of reliability in the same sub-scale.

We observed that the $12^{\text {th }}$ item in the Turkish version of the scale had higher convergent validity (AVE) under the roles and responsibilities sub-scale, as in Tamura et al.'s ${ }^{24}$ study; however, although this resulted in a small degree of improvement in the Chi-square test, RMSEA, and factor values, it was not included in the study because it did not result in any improvement in the AVE value. Similar results were observed for items 18 and 19. When high error values were considered, it was possible to exclude these items, as done by Cloutier et al. ${ }^{25}$; however, this exclusion decreased the degrees of freedom (df) and increased the $\mathrm{X}^{2}$ and RMSEA values. Therefore, we kept all 19 items in the scale (Table 5). The reliability coefficient in RIPLS and its adapted versions yields different results in various cultures. The sub-scale that has the most consistent structure was teamwork and collaboration. On the other hand, the roles and responsibilities sub-scale can be seen as the most problematic one. Different studies show inconsistent results for the professional identity sub-scale. ${ }^{21-23,26}$

The most outstanding results among the adaptation studies were obtained by Cloutier et al..$^{25}$ The $18^{\text {th }}$ and $19^{\text {th }}$ items in that study were excluded, and the remaining items were associated with different, re-named factors. The titles of the sub-scales and their associations with the factors are different in the Japanese ${ }^{24}$ and the French $^{25}$ adaptations of the original scale; when those adaptation studies were investigated, it was noted that an approach that considered cultural differences and subject fields was necessary to obtain similar results to the original scale. This approach resulted in differentiation in the type of factor analysis, the number of factors, and factor titles, as well as the distribution of items within the factors. This finding indicates that the results obtained from the scale can be affected by cultural differences, thus yielding different results. It is, therefore, imperative to take cultural variations into consideration when using the RIPLS and comparing the results. Cultural differences made it necessary to add wording to scale items. The expert panel in the Turkish adaptation study was required to make some changes in the items in order to make them more understandable (Items 10, 11, 16, 17, and 18). Similar efforts were made during the Japanese and French adaptation studies. This was reported in the Japanese study, specifically that "the Japanese translation included the context of learning in inter-professional groups". ${ }^{24}$ Tamura thought that the students could react to consecutive negative statements, because in Asian and Eastern cultures, expression of negative statements is seen as a sensitive matter. The efforts of the Turkish expert panel to change item 11 are a reflection of similar concerns as those of Tamura. Different statements can provoke different sensitivities between cultures. For example, in the Turkish adaptation study, the statement "the function of other healthcare professions is mainly to provide support for doctors", was perceived negatively by a non-physician member of the expert panel, indicating it was an insult to his profession. This problem was dealt with via detailed explanation of the reasons for the inclusion of both the positive and negative statements in the scale. For this reason, it is recommended that a standardized introduction explaining the purpose of the scale and the statements used in it 
Table 1: The descriptive characteristics and RIPLS scores of the students

\begin{tabular}{|c|c|c|c|c|c|c|}
\hline Professions & Year & Total & Mean & sd & Min. & Max. \\
\hline Paramedic & 2 & 17 & 74.53 & 8.66 & 58 & 87 \\
\hline Nutrition and Dietetics & $3-4$ & 70 & 83.27 & 6.78 & 57 & 92 \\
\hline Child Development & $2-3$ & 86 & 81.27 & 6.80 & 62 & 94 \\
\hline Dentistry & 3 & 56 & 75.33 & 7.03 & 58 & 88 \\
\hline Pharmacy & $3-4$ & 66 & 82.61 & 8.05 & 55 & 95 \\
\hline Physiotherapy and Rehabilitation & $2-4$ & 61 & 77.10 & 9.27 & 55 & 91 \\
\hline Nursing & $2-3$ & 61 & 78.26 & 7.12 & 59 & 92 \\
\hline Medicine & 4 & 67 & 69.38 & 9.46 & 45 & 94 \\
\hline Total & & 484 & 78.11 & 9.39 & 45 & 95 \\
\hline
\end{tabular}

Table 2: Confirmatory factor analysis: reliability, validity and fit indices of three factors model

\begin{tabular}{|c|c|c|c|c|c|c|c|c|c|c|c|c|c|}
\hline & & \multicolumn{4}{|c|}{ Reliability - validity } & \multicolumn{8}{|c|}{ Index fit } \\
\hline Indicator & a & CR & AVE & MSV & ASV & df & $x^{2}$ & RMSEA & GFI & AGFI & RMR & SRMR & $\mathrm{NNFI}$ \\
\hline $\begin{array}{c}\text { Three Factors } \\
\text { Model }\end{array}$ & 0.87 & & & & & 149 & 445.98 & 0.064 & 0.91 & 0.89 & 0.043 & 0.049 & 0.97 \\
\hline $\begin{array}{l}\text { Teamwork and } \\
\text { Collaboration }\end{array}$ & 0.85 & 0.87 & 0.42 & 0.71 & 0.36 & & & & & & & & \\
\hline $\begin{array}{l}\text { Professional } \\
\text { Identity }\end{array}$ & 0.82 & 0.85 & 0.45 & 0.14 & 0.43 & & & & & & & & \\
\hline $\begin{array}{c}\text { Roles and } \\
\text { Responsibilites }\end{array}$ & 0.28 & 0.34 & 0.20 & 0.06 & 0.10 & & & & & & & & \\
\hline
\end{tabular}

CR: Composite Reliability, AVE: Average Variance Extracted, MSV: Maximum Shared Variance, ASV: Average Shared Variance, RMSEA: Root Mean Error of Approximation, GFI: Goodness of Fit Index, AGFI: Adjusted GFI, RMR: Root Mean Square Residual, SRMR: Standardized RMR, NNFI: Non-Normed Fit Index.

\begin{tabular}{|c|c|c|c|}
\hline \multicolumn{3}{|c|}{ Table 3: Pearson Correlation and Average Variance Extracted (AVE) Matrix of Subscales } \\
\hline & Teamwork & Professional Identity & Roles \& Responsibilities \\
\hline Teamwork & $0.42^{\mathrm{a}}$ & & \\
\hline Professional Identity & $0.84^{\mathrm{b}}(0.71)^{\mathrm{c}}$ & $0.45^{\mathrm{a}}$ & \\
\hline Roles \& Responsibilities & $0.24^{\mathrm{b}}(0.06)^{\mathrm{c}}$ & $0.37^{\mathrm{b}}(0.14)^{\mathrm{c}}$ & $0.20^{\mathrm{a}}$ \\
\hline
\end{tabular}

$\mathrm{a}=\mathrm{AVE}, \mathrm{b}=$ Pearson Correlation, $\mathrm{c}=$ Pearson Correlation ${ }^{2}$

\begin{tabular}{|c|c|c|c|c|c|c|c|}
\hline Test & Desired Value & $\begin{array}{c}\text { Parsell } \\
\text { 1999*}\end{array}$ & $\begin{array}{l}\text { McFadyen } \\
2005\end{array}$ & $\begin{array}{c}\text { Lauffs } \\
2008\end{array}$ & $\begin{array}{c}\text { Tamura } \\
2012\end{array}$ & $\begin{array}{c}\text { Tyastuti } \\
2014\end{array}$ & Turkish \\
\hline Normed Chi-square (x2/df) & $<3$ & 2.154 & 1.777 & & & 2.48 & 2.99 \\
\hline Goodness of Fit Index (GFI) & $>0.9$ & 0.902 & 0.904 & & & 0.92 & 0.91 \\
\hline Comparative Fit Index (CFI) & $>0.9$ & 0.872 & 0.942 & 0.95 & 0.93 & 0.94 & 0.97 \\
\hline Normed Fit Index (NFI) & $>0.9$ & & & & & 0.91 & 0.96 \\
\hline Non-Normed Fit Index (NNFI) & $>0.95$ & & & 0.95 & & & 0.97 \\
\hline Root Mean Error of Approximation (RMSEA) & $<0.08$ & 0.061 & 0.054 & 0.037 & 0.06 & 0.063 & 0.064 \\
\hline
\end{tabular}

*adapted by McFadyen et al. ${ }^{22}$ 
be provided as part of the study. Furthermore, reporting similar events encountered in other adaptations and comparative studies would be useful.

As the number of professions enrolled in the study increased, the efforts to make the items more inclusive also increased. The scale was studied in different healthcare professions in different studies. Some studies did not enrol representatives from professions like medicine, dentistry, and pharmacy. Similarly, there are variances observed in the variables, such as the number of years of study for the students, their professional identities, the number of participants, and the years from graduation. These variances in the development of the scale should be considered when conducting intercultural comparative studies.

We did not include first-year healthcare students (Table 1) in the present study in order to overcome the limitation of experience, as mentioned in the previous studies. Parsell and Bligh ${ }^{21}$ stated that undergraduate students cannot properly evaluate the sub-scales due to a lack of experience. Similarly, McFadyen et al. ${ }^{22}$ reported that judgments about the professional roles and responsibilities of the novice students were not fully developed. We think it is an important point to consider. On the other hand, students have their judgments influenced as they get to know other healthcare students. For instance, during the pilot study of the Turkish version of the RIPLS, a medicine student expressed a need to add 'excluding dentists and pharmacists' to the $17^{\text {th }}$ statement. Researchers are cautious to perform sub-factor comparison during adaptation studies due to the different validity and reliability values obtained from different studies. It can be seen that the same recommendation is also valid for the Turkish version of RIPLS; on the other hand, when the scale is considered as a single dimension, the measurement tools obtained from the adaptation studies yield similar results by producing similar Cronbach's Alpha values for single-dimension professional readiness measurements. ${ }^{21-23,25,27}$

\section{CONCLUSION}

This study was conducted to translate and validate the Turkish version of the RIPLS. The results indicated that the psychometric properties of the adapted scale were comparable to those of the original English version. The Turkish RIPLS had the lowest value of reliability for the 'roles and responsibilities' subscale, like the original and the other adapted scales. Considering that all translated or adapted scales measured one main factor, named the readiness, they had consistent results; however, adaptation studies showed that the RIPLS was sensitive to cultural context, mainly in the defining of subscales. Researchers should be cautious on performing cross-cultural comparisons of subscale 'roles and responsibilities'.

The single-time responses of 484 volunteer-samples from some disciplines may not represent the evidence on the stable responses of the entire Turkish student population in healthcare education; it does not support for generalization and standardization needs, such as serving as a standardized evaluation tool. Discrimination capacity of the assessment tool for detecting differences of quality educational programs should be studied.

We suggest some future research with the healthcare students using the RIPLS at our university: a study evaluating the various groups of students who attended versus those who did not attend the elective course, a pre/post evaluation of the students' readiness before and after attending the inter-professional learning modules or courses, and a longitudinal study to evaluate students' readiness from freshman year through graduation.

Considering the conformity to the original model and psychometric performance, the Turkish RIPLS can be used as a valid and reliable measurement tool for evaluating the interprofessional learning readiness of healthcare students.

\section{CONFLICT OF INTERESTS}

The author(s) declared no potential conflicts of interest with respect to the research, authorship, and/or publication of this article.

\section{ACKNOWLEDGEMENT}

We especially thank AK McFadyen for his contributions to the development of the article.

\section{REFERENCES}

1. World Health Organization. Framework for action on inter-professional education and collaborative practice. WHO study group on Inter-professional Education and Collaborative Practice, Genova: World Health Organisation Press. 2010.

2. World Health Organization. Learning together to work together for health: report of a WHO Study Group on Multi professional Education of Health Personnel: the Team Approach, Geneva: World Health Organisation Press. 1988.

3. World Federation for Medical Education. Basic Medical Education: WFME Global Standards for Quality Improvement, Copenhagen: WFME OFFICE. 2003.

4. De Vito KM. Implementing adult learning principles to overcome barriers of learning in continuing higher education programs. Online Journal for Workforce Education and Development. 2010;3(4):1.

5. Headrick LA, Wilcock PM, Batalden PB. Inter-professional working and continuing medical education BMJ. 1998;316(7133):771-4. https://doi. org/10.1136/bmj.316.7133.771 PMid:9529419 PMCid:PMC1112732. 
6. Kohn LT, Corrigan JM, Donaldson MS, editors. To err is human: building a Safer Health System. Washington: National Academies Press. 2000.

7. Baker GR, Norton PG, Flintoft V, Blais R, Brown A, Cox J, et al. The Canadian Adverse Events Study: the incidence of adverse events among hospital patients in Canada. Can Med Assoc J. 2004;170(11):1678-86. https://doi. org/10.1503/cmaj.1040498 PMCid:PMC408508.

8. Ornato JP, Peberdy MA, Reid RD, Feeser VR, Dhindsa HS, NRCPR Investigators. Impact of resuscitation system errors on survival from in-hospital cardiac arrest. Resuscitation. 2012;83(1):63-9. https://doi. org/10.1016/j.resuscitation.2011.09.009 PMid:21963583.

9. World Health Organization. Patient safety curriculum guide: multi-professional edition. WHO Patient Safety. Geneva: World Health Organisation Press. 2011

10. Morey JC, Simon R, Jay GD, Wears RL, Salisbury M, Dukes KA, et al. Error reduction and performance improvement in the emergency department through formal teamwork training: evaluation results of the Med Teams project. Health Serv Res. 2002;37(6):1553-81. https://doi.org/10.1111/14756773.01104 PMid:12546286 PMCid:PMC1464040.

11. Hammick M, Freeth D, Koppel I, Reeves S, Barr H. A best evidence systematic review of interprofessional education: BEME Guide no. 9. Med Teach. 2007;29(8):735-51. https://doi.org/10.1080/01421590701682576 PMid:18236271.

12. World Health Organization. Human factors in patient safety: Review of topics and tools. Report for Methods and Measures, Working Group of WHO Patient Safety. Geneva: World Health Organisation Press; 2009.

13. Herbert CP. Changing the culture: Inter-professional education for collaborative patient-centred practice in Canada. J Inter prof Care. 2005;19:1-4. https://doi.org/10.1080/13561820500081539 PMid:16096140.

14. Barr H, Waterton S. Summary of a CAIPE survey: inter-professional education in health and social care in the United Kingdom. J Interprof Care. 1996;10(3):297-303. https://doi.org/10.3109/13561829609034117.

15. Barr H, Hammick M, Koppel I, Reeves S. Evaluating inter-professional education: two systematic reviews for health and social care. Br Educ Res J. 1999;25(4):533-44. https://doi.org/10.1080/0141192990250408.

16. Clark PG. What would a theory of inter-professional education look like? Some suggestions for developing a theoretical framework for teamwork training. J Interprof Care. 2006;20(6):577-89. https://doi. org/10.1080/13561820600916717 PMid:17095437.

17. Oandasan I, Reeves S. Key elements of inter-professional education. Part 2: Factors, processes and outcomes. J Inter prof Care. 2005;19(1):39-48. https://doi.org/10.1080/13561820500081703 PMid:16096144.

18. A national inter-professional competency framework. Canadian Interprofessional Health Collaborative Competencies Working Group. Vancouver: University of British Columbia. 2010.

\section{PICTORIAL ABSTRACT}

Turkish RIPLS

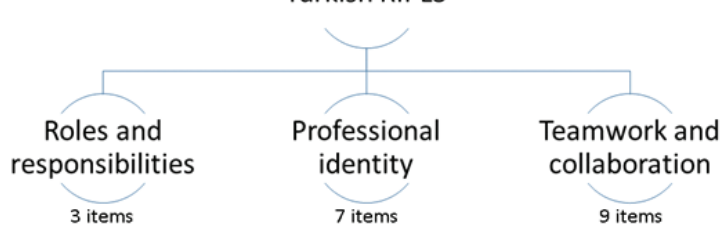

19. Core competencies for inter-professional collaborative practice: Report of an Inter-professional Education Collaborative Expert Panel. Washington D.C: Inter-professional Education Collaborative. 2011.

20. Lapkin S, Levett-Jones T, Gilligan C. A systematic review of the effectiveness of inter-professional education in health professional programs. Nurse Educ Today. 2013;33(2):90-102. https://doi.org/10.1016/j.nedt.2011.11.006 PMid:22196075

21. Parsell G, Bligh J. The development of a questionnaire to assess the readiness of health care students for inter-professional learning (RIPLS). Med Educ.1999;33(2):95-100. https://doi.org/10.1046/j.1365-2923.1999.00298.x PMid:10211258

22. McFadyen AK, Webster V, Strachan K, Figgins E, Brown H, McKechnie J. The Readiness for Inter-professional Learning Scale: A possible more stable sub-scale model for the original version of RIPLS. J Inter prof Care. 2005;19(6):595-603. https://doi.org/10.1080/13561820500430157 PMid:16373215

23. Lauffs M, Ponzer S, Saboonchi F, Lonka K, Hylin U, Mattiasson AC. Cross-cultural adaptation of the Swedish version of Readiness for Interprofessional Learning Scale (RIPLS). Med Educ. 2008;42(4):405-11. https:// doi.org/10.1111/j.1365-2923.2008.03017.x PMid:18338993.

24. Tamura Y, Seki K, Usami M, Taku S, Bontje P, Ando H, et al. Cultural adaptation and validating a Japanese version of the readiness for interprofessional learning scale (RIPLS). J Inter prof Care. 2012;26(1):56-63. https://doi.org/10.3109/13561820.2011.595848 PMid:22233369.

25. Cloutier J, Lafrance J, Michallet B, Marcoux L, Cloutier F. French translation and validation of the Readiness for Inter-professional Learning Scale (RIPLS) in a Canadian undergraduate healthcare student context. J Inter prof Care. 2015;29:150-5. https://doi.org/10.3109/13561820.2014.942837 PMid:25076020.

26. Mahler C, Rochon J, Karstens S, Szecsenyi J, Hermann K. Internal consistency of the readiness for inter-professional learning scale in German health care students and professionals. BMC Med Educ. 2014;14(1):1. https:// doi.org/10.1186/1472-6920-14-145 PMid:25027384 PMCid:PMC4107476.

27. Tyastuti D, Onishi H, Ekayanti F, Kitamura K. Psychometric item analysis and validation of the Indonesian version of the Readiness for Inter-professional Learning Scale (RIPLS). J Inter prof Care. 2014;28(5):426-32. https://doi.org /10.3109/13561820.2014.907778 PMid:24731139.

28. Sousa VD, Rojjanasrirat W. Translation, adaptation and validation of instruments or scales for use in cross-cultural health care research: a clear and user-friendly guideline. J Eval Clin Pract. 2011;17(2):268-74. https://doi. org/10.1111/j.1365-2753.2010.01434.x PMid:20874835.

29. Chambers RL. Outlier robust finite population estimation. J Am Stat Assoc. 1986;81(396):1063-9. https://doi.org/10.1080/01621459.1986.10478374

\section{SUMMARY}

- New educational modalities like "Inter-professional collaboration for patient safety and improvement the quality of care" requires measuring tools to determine the qualifications of the learners and support the learning process.

- This study aims to adapt the readiness for interprofessional learning scale (RIPLS) for use in Turkey.

- New Turkish RIPLS is consistent with the original scale. It can be used as a valid and reliable measurement tool for evaluating the RIPL.

- The RIPLS is sensitive to cultural context. Researchers should be cautious on performing cross-cultural comparisons of subscale 'roles and responsibilities'. 


\section{About Authors}

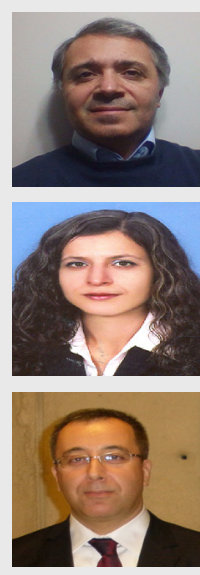

Dr. Arif Onan: He is presently working as Educational Technologist and Simulation Specialist at the Department of Medical Education and Informatics. His studies mainly focused on the facilitating learning and improving performance via selecting and using appropriate technology.

Dr. Sevgi Turan: Is presently working as associate professor at the Department of Medical Education and Informatics. Her studies mainly focused on the curriculum development, learning skills and health science education.

Dr. Melih Elcin: Has been working as the head of the Department of Medical Education and Informatics. He is the current president of Turkish Association for Medical Education. His areas of interest are program development, evaluation, clinical and communication skills training, standardized patients, simulation, interprofessional education and medical humanities. He is the member of ASPE, SESAM and SSH.

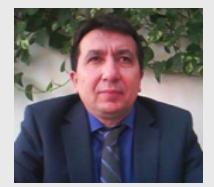

Dr. Nurettin Șimșek: Is presently working as Head of Department of Computer Education \& Instructional Technologies at the Faculty of Educational Sciences. He is currently President and Manager of National Association for Educational Science \& Practices. and a member of Distance Education Commission Higher Education Council (YÖK).

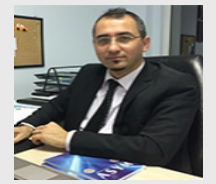

Dr. Kaan Zülfikar Deniz: Is presently working as manager of Ankara University Testing Center. He has experience in the area of assessment and evaluation, development of measurement tools. He has given master and doctoral courses about multivariate statistics, statistic and scientific research methods.

Cite this article: Onan A, Turan S, Elcin M, Simsek N, Deniz KZ. A Test Adaptation of the Modified Readiness for Inter-professional Learning Scale in Turkish. Indian J of Pharmaceutical Education and Research. 2017;51(2):207-15. 